\title{
De la valoración racional de la prueba en la verdad procesal a la teoría de la probabilidad preponderante ${ }^{1}$ From the rational appraisal of evidence in procedural truth to the theory of preponderant probability
}

\author{
DOI: http://dx.doi.org/10.17981/juridcuc.14.1.2018.12
}

Artículo de investigación. Fecha de recepción: 20/08/2018 Fecha de aceptación: 01/11/2018

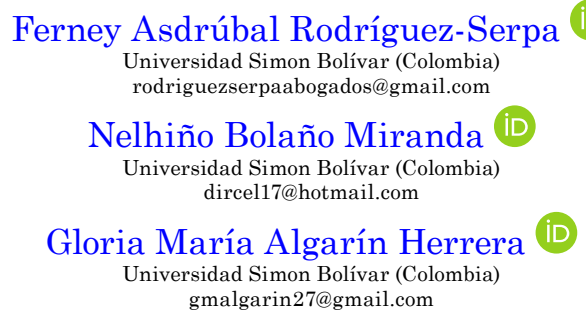

Para citar este artículo:

Rodríguez, F., Bolaño, N. y Algarín, G. (2018). De la valoración racional de la prueba en la verdad procesal a la teoría de la probabilidad preponderante. JURÍDICAS CUC, vol. 14, no. 1, pp. 259-286. DOI: http://dx.doi.org/10.17981/juridcuc.14.1.2018.12

\begin{abstract}
Resumen
El presente artículo aborda la categoría de la prueba vinculada a la perenne búsqueda de hallar la verdad absoluta por parte del Estado jurisdicción en su esfuerzo epistemológico de valoración racional de la prueba de los hechos. No obstante, desde el método inductivo, el presente trabajo tuvo por objeto determinar - muy a pesar de las infinitas dificultades de alcanzar tal pretensión- los desarrollos doctrinales que convergen en la misión por alcanzar una verdad relativa, o verdad procesal que abandona el esfuerzo por la verdad verdadera, la cual, a pesar de las dificultades cognoscitivas, se demuestra en este estudio cualitativo que esto sí es posible (verosímilmente) a través de la teoría de la probabilidad preponderante, lo cual lleva, en efecto, a concluir que la verdad de los hechos puede reconstruirse a través de los medios de prueba, y con ello, alcanzar una verdad relativa reforzada en grados bajo los esquemas de probabilidad preponderante. Palabras clave: prueba, verdad, valoración racional, verdad procesal, teoría de la probabilidad
\end{abstract}

\section{Abstract}

This article deals with evidence as linked to the perennial search to find the absolute truth on the part of the State's jurisdiction in its epistemological effort for rational evaluation of the evidence of facts. However, from the inductive method, the present work had the purpose of determining -in spite of the infinite difficulties of reaching such a pretension- the doctrinal developments that converge in the mission to reach a relative truth, or a procedural truth that abandons the effort for the true truth. Regardless of the cognitive difficulties, this qualitative study demonstrates that this is possible through the theory of the preponderant probability, which leads to the conclusion that the truth of the facts can be reconstructed through the means of evidence and, with it, reach a relative truth reinforced in degrees under the preponderant probability schemes.

Keywords: Test, truth, rational assessment, procedural truth, probability theory

\footnotetext{
${ }^{1}$ Artículo derivado del proyecto de investigación titulado "La racionalidad de la prueba en el marco de la teoría de la probabilidad", desarrollado por el grupo de investigación "Derechos humanos, tendencias jurídicas y sociojuridicas contemporáneas" de la Universidad Simón Bolívar de Barranquilla (Colombia).
}

The author; licensee Universidad de la Costa - CUC. 


\section{Introducción}

La historia de los diversos procesos ante la jurisdicción ha estado investida por la dicotomía teleológica de pacificar conflictos inter-parte e ir más allá de estos para lograr reconstruir la verdad de los hechos históricos, y, en consecuencia, lograr satisfacer la justicia rogada por alguno de los intervinientes legítimos en un proceso. Tal tensión, sin embargo, ha sido una victoria a favor de la verdad y, para ello, la prueba ha resultado ser la mayor garantía para la reconstrucción de los hechos del pasado, es decir, se ha constituido epistemológicamente en el vehículo de conocimiento más sólido y con más certeza para alcanzar la verdad.

No obstante, todos estos no han sido lineales. Todo ha estado minado de disimiles crisis empíricamente registradas - por los infinitos abusos del poder judicial-, so pretexto de conseguir instrumentalmente la verdad. Con ello se hace referencia a la tortura, que en algún momento de la historia era el principal instrumento para lograr la verdad, es decir, una verdad alcanzada a través de medios injustos o una verdad reconstruida para satisfacer caprichos, lo cual en efecto no podría ser verdad.

El punto de ruptura entre la barbarie de la tortura y la práctica razonable de la prueba se determina por el nacimiento del Estado de Derecho, el cual resulta ser el giro determinante que puso fin a la toma de decisiones arbitrarias basadas en la íntima convicción del juez, para pasar a decisiones judiciales donde el valor de la prueba de los hechos lo determinaba el legislador racional.

En el siglo XX, y lo que va de este, el valor de la prueba ha sido más diáfano en cuanto al diseño de los medios de pruebas y, con ello, se ha llegado plenamente a la doctrina de la verdad procesal. Sin embargo, ésta, más allá de los constructos teóricos, no es garantía de una verdad absoluta, de ahí los escépticos sobre los alcances de la prueba y las imposibilidades de llegar a la verdad. La respuesta al escepticismo ha sido que sin duda existe una verdad, pero no absoluta: una verdad relativa, lo cual no es una condición suficiente para saciar las demandas de verdad frente a los hechos. 
Ante tal insuficiencia, ha emergido desde la entrañas del mundo cuantitativo, pero con una lectura cualitativa de la prueba, una teoría que más que emergente, es una teoría para quedarse, se trata de la teoría de la probabilidad preponderante, con la cual se estima que si es imposible llegar a la verdad, entonces se debe hacer el esfuerzo de llegar a ella, o sea, que no se descanse hasta lograr lo más verosímil de la verdad de los hechos y lograr la mayor certeza para derrotar cualquier estándar de prueba, ya sea éste el de probabilidad prevalente o el de más allá de toda duda razonable.

Para ampliar la comprensión de este trabajo, se pretende en un primer momento mirar la relación entre la prueba y la valoración racional de la prueba, donde no solo se estudia la prueba como categoría, sino también en sus partes, como fuente, medio y objeto de prueba; en un segundo momento se estudia la verdad procesal, sus alcances y penurias; y finalmente, se realizará una aproximación a la teoría de la probabilidad con superación de la verdad procesal.

\section{La prueba y su valoración racional}

En todo escenario de discusión jurídica es ineludible abocarse a la prueba (Vega, 2015, p. 43), la cual conduce a la acreditación de la certeza de un hecho en el que el ser humano, por naturaleza, siempre está a la expectativa de recrear o reconstruir los hechos históricos, esto es, de encontrar evidencias que soporten la ocurrencia o no de tales o cuales afirmaciones sobre lo acaecido. Para ello, no solo basta su aporte como sujetos racionales, también es esencial la cientificidad para el registro confiable empíricamente del aporte de las partes y de la valoración de confirmación del juez. Por ello, como acota Vargas (2011): "La ciencia jurídica en muchos aspectos utiliza la palabra hecho, y en general la prueba es para verificar hechos relacionados con las alegaciones de las partes" (p. 143).

Ampliando lo expuesto, Tirado (2011) manifiesta que "la prueba en derecho es la 'sustancia' por medio de la cual se accede a la 'victoria' en los procesos judiciales (...) de quien logró 
'probar': quien tenía legitimidad y razón en la disputa jurídica a partir de que el interesado haya logrado 'probar' que tenía consigo la 'verdad', y este es precisamente el problema de la justicia” (p. 16).

Problema que epistemológicamente no es nada fácil de resolver, pues la prueba por sí sola no habla, se necesita de la intervención racional del tercero supra ordenado, garante de la tutela judicial efectiva, es decir, del Estado jurisdicción (Rodríguez y Ospino, 2009), el cual ha de tener la capacidad casi infinita de interpretar y valorar la ocurrencia de los hechos históricos a través de los medios de pruebas facilitados por las partes o impulsados por el mismo.

De ahí, la importancia del rol del Estado jurisdicción o del juez, ya que éste también cumple con una función creadora de Derecho, donde:

(...) podemos inferir que el proceso de producción y aplicación del Derecho está íntimamente vinculado con la función del juez, quien al mismo tiempo forma parte del mismo, al ser el elemento humano a quien corresponde decidir sobre los hechos alegados por las partes en un juicio, teniendo la extraordinaria tarea de dirimir un conflicto sobre la base de normas adjetivas y sustantivas y muy especialmente de lo que los legitimados activo y pasivo hubiesen probado (Acosta, 2007, p. 53).

De regreso a la tutela judicial efectiva, ésta está intrínsecamente vinculada a la naturaleza de derecho fundamental, tal y como se puede ver de lo prescrito por Ruiz (2007):

El derecho fundamental a la prueba se entiende como un derecho subjetivo en el que el ordenamiento jurídico crea una posición jurídica de un sujeto de exigir la prueba frente a otro-el juez- quien actúa como obligado. Esta exigencia, de conformidad con el inc. 4 del Art. 29 de la $\mathrm{CP}$, consiste en la presentación de pruebas y en la contradicción de las que se alleguen en su contra en aras de propender por el interés material que se demanda o que se defiende (p. 186). 
Por esta razón, la prueba despliega como institución una carga de garante de los derechos fundamentales, que no es otra cosa que la protección del derecho material subjetivo de una de las partes, pero, a su vez, tal consideración ha de ser flexible pues ha de tener la posibilidad de ser dúctil en el contradictorio, que no es solo un derecho de la contra parte, ha de ser también un derecho de naturaleza fundamental.

También puede valorarse como el conjunto particular de recursos que pueden utilizarse para obtener aquella demostración, es decir, de ver la prueba desde la racionalidad instrumental o como medio. Para ello es importante la inmediación del juez, quien dirime el conflicto teniendo en cuenta las pruebas aportadas al proceso. A semejante responsabilidad le deben asistir, entre otros principios, el de racionalidad, que puedan servir como guía orientadora de la correcta decisión judicial (Rodríguez, 2009).

Para llegar a la correcta decisión judicial, es menester analizar en minucia el concepto de prueba como fuente, medio y objeto de la prueba. En cuanto al primero, la fuente de la prueba, ha de ser la existencia real de la ocurrencia del hecho que ha dejado datos brutos que requieren ser extraídos para asignarles un significado a sus contenidos materiales; por su parte, el medio de prueba hace referencia a aquellas vías permitidas por la ley para poder probar los hechos; y con relación a y por objeto de la prueba se entiende el hecho, cosa o circunstancia que se debe probar.

En este sentido, sin referirse al objeto de la prueba, Meneses (2008) afirma que la fuente de prueba corresponde al escenario previo y ajeno al proceso, en tanto los medios de prueba pertenecen de lleno al campo judicial, es decir, al proceso, a lo que ocurre al interior de él. Para Carnelutti (1982, citado por, Acosta, 2007), la fuente se define como: "los hechos percibidos por el juez y que le sirven para la deducción del hecho a probar" (p. 58). Al respecto del concepto dado por el italiano Carnelutti sobre la fuente de prueba, manifiesta Acosta (2007) lo siguiente: 
A su juicio las fuentes de las pruebas se agrupan en dos grandes categorías según constituyan o no la representación del hecho a probar, pero dejando por sentado que en cualquiera de los dos casos existen fuentes de la prueba. En tal sentido, este autor distingue entre las fuentes de prueba constituidas por la representación del hecho a probar; y las fuentes de presunción que son todos aquellos hechos que sirven para la deducción del hecho a probar y que no están constituido por la representación de éste (p. 58).

En cuanto a los medios de prueba, como aquellos que sirven de instrumento para acercar al juez con los hechos del pasado, y según Alsina (1958, citado por Acosta, 2007), se entienden como el instrumento, cosa o circunstancia en los que el juez encuentra los motivos de su convicción (p. 60).

Esto explica por qué el medio de prueba es mayúsculo para la comprensión del ejercicio de valoración probatoria preponderante por parte del juez y, en consecuencia, de las partes en el proceso. En esta dimensión "los medios de prueba se sitúan en el área del proceso. Tratase del conjunto de elementos que se aceptan en una causa para acreditar las afirmaciones de hecho en torno a las cuales debaten las partes ante el tribunal" (Meneses, 2008, p. 16).

En cuanto al objeto de la prueba, este hace referencia a la demostración de un hecho. En palabras de Acosta (2007), también hace referencia a la "inexistencia de un hecho, por lo tanto, todo lo que pueda ser objeto del conocimiento y que se alega como fundamento del derecho que se pretende, debe ser entendido como objeto de la prueba” (p. 62). En relación a esto, Echandía (1984, citado por Acosta, 2007) sostenía que, "la prueba debe entenderse lo que se puede probar en general, es decir, resulta aquello sobre lo que puede recaer la prueba y que sea susceptible de comprobación ante el órgano jurisdiccional del Estado, abarcando los hechos pasados, presentes y futuros" (Acosta, 2007, p. 63).

Lo anterior explica que sin hechos no existiría razón de ser de la prueba puesto que no existe supuesto de hecho perseguible para obtener el efecto consagrado en la norma de calificar el hecho en el supuesto fáctico de ella, labor que ha de demandar la participación del juez en la valoración racional de la prueba 
y, en efecto, en la adjudicación epistemológica de reconstruir el hecho en razón del supuesto fáctico de las normas materia de análisis en el caso concreto suscitado.

En esta lógica, Vargas (2011) establece que "la actividad probatoria y la prueba judicial, en su sentido de conocimiento de la existencia o inexistencia de un hecho, son una creencia racional y fundada del juez sobre el hecho" (p. 144), lo cual es cierto, pero no de cualquier forma ha de ser racional, alejada de la simplicidad y caprichos del juzgador.

Conocida así las distinciones de fuente, medio y objeto de prueba, es necesario establecer la pertinencia de ésta por parte del juez, el cual siempre ha de tener al frente la dicotomía de decretar su pertinencia o señalar todo lo contrario. En coherencia con ello, Verdugo (2010) manifiesta que la pertinencia:

es un examen de carácter lógico que deja de lado la pregunta sobre la credibilidad de la prueba, limitándose a responder la siguiente pregunta: si la prueba fuese presentada y creída en el juicio, la información que contiene ¿sirve para hacer más posible que los hechos hayan ocurrido de la manera que sostiene la parte que la ofrece? Si la respuesta es afirmativa, la prueba es pertinente y debe ser admitida. Si la respuesta es claramente negativa, la prueba es impertinente y debe ser excluida. Si la respuesta no es clara, la prueba debe ser admitida ya que debe favorecer a la parte que la presenta pues ella tiene mayor conocimiento de su caso y tiene el derecho subjetivo a presentar prueba (p. 149).

La pertinencia de la prueba no es la única condición que debe cumplir la prueba para ser admitida, debe evitar ser excluida por alguna de las otras causales de exclusión establecidas en la ley, como la prueba obtenida por medios ilícitos, los medios de prueba sobreabundantes o la que recaiga sobre hechos públicos y notorios (Verdugo, 2010, p. 150).

Todo esto declara, que la valoración racional de la prueba evidentemente está en manos del Estado jurisdicción, quien establece tres criterios, según Meneses (2008), "un juicio jurisdiccional como 'saber-poder', vale decir, una visión donde la tarea del órgano jurisdiccional es una combinación entre conocimiento del conflicto (veritas) y decisión del mismo (auctoritas)" (p. 24). 
Otra de las cuestiones que ha de establecer el juez en su labor racional se relaciona con la noción semántica de verdad procesal fáctica, que se comprende como "correspondencia" entre lo dicho al interior del proceso por las partes en conflicto y lo que ocurrió en el hecho histórico. Cuestión esta que no es nada fácil por las limitaciones desde la condición humana que tiene el juez, pero que no por ello renuncia a la posibilidad de aproximarse a la realidad a partir de las inferencias racionales que se derivan de los antecedentes que se tienen en determinado momento y lugar (Meneses, 2008, p. 24).

$\mathrm{Y}$, por último, se asocia en sentido estricto a la prueba el juez, quien participa de una "actividad tendiente a aprehender y, en la mayoría de los casos, reconstruir hechos efectivamente acaecidos, a partir de una valoración judicial de la suficiencia de la información que proporciona cada uno de los medios acompañados a la causa" (Meneses, 2008, p. 25). De ahí, como se ha dicho por disimiles de doctrinantes, "la finalidad central de la prueba es cognoscitiva, como quiera que está encaminada a obtener un conocimiento probable de los hechos" (Meneses, 2008, p. 25).

No solo Meneses (2008) establece criterios frente a la prueba, también lo hace el tratadista italiano Taruffo (2005), quien cimienta los criterios de la elección racional de la prueba en cuatro premisas, a saber, relacionadas con el estándar de prueba de probabilidad prevalente. Sobre tales criterios aporta:

a) Que se conciba la decisión del juez sobre los hechos como el resultado final de elecciones en torno a varias hipótesis posibles relativas a la reconstrucción de cada hecho de la causa; b) Que estas elecciones se conciban como si fueran guiadas por criterios de racionalidad; c) Que se considere racional la elección que toma como "verdadera" la hipótesis sobre hechos que resulta mejor fundada y justificada por las pruebas respecto a cualquier otra hipótesis; d) Que se utilice, como clave de lectura del problema de la valoración de las pruebas, no un concepto genérico de probabilidad como mera no certeza, sino un concepto específico de probabilidad como grado de confirmación de la veracidad de un enunciado sobre la base de los elementos de confirmación disponibles (p. 1298). 
En todo caso, es importante, dejar en claro la idea de racionalidad que ha de tenerse para controlar la valoración de la prueba que se materializa en la decisión judicial cuando esta se construye a partir de materiales externos y perceptibles que entreguen noticias de los acontecimientos, y exige la concurrencia de elementos controlables y verificables mediante criterios intersubjetivos (Meneses, 2008, p. 28) de las pruebas aportadas por los sujetos procesales. Todo esto equivale a decir que el juez tiene el deber preciso de extraer, de su contacto directo con la prueba, "los factores epistémicamente aceptables (...), donde debe construir inferencias racionales, fundadas sobre reglas o estándares de valoración que deben ser claramente identificables, sobre todo por el propio juez que los usa" (Taruffo, 2008, p. 26).

En esta misma línea, indica Vargas (2010),

la valoración de la prueba está presente en todo proceso jurisdiccional. Es un momento último con el cual culmina la llamada actividad probatoria, que consiste en una operación epistemológica que realiza el juez y cuya finalidad estriba en establecer el mérito o convicción que puede inferirse del material probatorio allegado al proceso. Por lo tanto, determinará la eficacia que las fuentes de prueba incorporadas al proceso mediante los medios de prueba, y establece con ello, la importancia en la formación de la convicción del Estado jurisdicción (p. 130).

Es evidente comprender que la racionalidad no solo descansa en la valoración de la prueba, sino en un lugar que dé cuenta de dicha valoración racional, que sin duda ha de ser el último acto jurisdiccional, la decisión judicial. Es aquí donde, "la motivación constituye una exposición del razonamiento justificativo de las bases en las cuales se fundamenta la decisión judicial" (Peña, 2008, p. 112). Es de agregar, que "tal exposición ha de efectuarse mediante argumentos que den cuenta de la eficacia atribuida a cada uno de los medios de prueba, del resultado de la evaluación global, y de la elección de la hipótesis fáctica asumida como verdadera reconstrucción de los hechos, en función del mayor grado de confirmación" (Peña, 2008, p. 112). 
En suma, "la argumentación, se expresa en la motivación de la sentencia, y corresponde a la justificación de las decisiones adoptadas, que ha de ser entendida en términos de aportar razones, lo cual se manifiesta como una explicitación del razonamiento efectuado por el juez" (Peña, 2008, p. 112) sobre la valoración de la prueba de los hechos.

\section{La verdad procesal}

Entendiéndose el proceso judicial como la búsqueda efectiva de justicia, se coligue que dentro de los fines y objetivos principales del mismo se enmarca la búsqueda de la verdad. Sin embargo, mucho se ha hablado respecto de la relatividad que engloba la verdad dentro de un proceso, pues gira en torno a la determinación del juez con respecto a los hechos y el material probatorio ofrecido en el juicio.

Puede entonces afirmarse que, teniendo en cuenta este enfoque, el proceso judicial en sí mismo se concibe como un método que propone el descubrimiento de la verdad y la reconstrucción de los hechos que dan cuenta de la verdad de las cosas. Ahora, esta verdad, denominada verdad procesal o jurídica, no llega a ostentar la calidad de absoluta, pues los hechos encuentran sus fundamentos en pruebas obtenidas durante el juicio.

En ese sentido, el juez a partir de la prueba cuenta con un instrumento idóneo para lograr reconstruir de un modo veraz los hechos que dieron origen al litigio a dirimir. De acuerdo con Taruffo (2005), en el proceso judicial solo se puede considerar como verdadero todo aquello que se prueba y solo en la medida en que tales pruebas disponibles ofrecen un apoyo cognoscitivo suficiente a los pronunciamientos de hecho. Es así como se afirma que la verdad procesal es totalmente relativa, pues precisamente depende de lo probado en el proceso (p. 1285).

En esencia, teniendo como objetivo final la resolución del conflicto o controversia, para Taruffo (2008) se deriva de ello que la justicia de la decisión es un factor determinante de la 
justicia del proceso, de modo que la verdad de los hechos se constituye como uno de los requisitos imprescindibles, que permiten llegar a una decisión judicial justa, entendiendo aquí la justicia en un sentido sustancial, es decir, justicia de los resultados del procedimiento.

Una vez dicho lo anterior, es menester definir ciertos conceptos que apoyen la labor de descripción de la verdad procesal. Primeramente, surge el término verdad, el cual para Ruiz (2016, p. 1) guarda una vinculación muy estrecha con el Derecho, especialmente en la solución de conflictos llevada a cabo a través del proceso judicial, en el sentido de que el juez cuando resuelve la controversia jurídica que ha conocido, tiene como base el conocimiento sobre la verdad de las proposiciones referidas a los hechos controvertidos, y adicionalmente, el conocimiento dogmático del Derecho.

De otra parte, se cuestiona el concepto conjugado de verdad, de si es necesario o no discutirse sobre la verdad para el mundo jurídico, especialmente en el ámbito del derecho procesal, pues, tal y como evidencia Taruffo, se asiste en los últimos decenios a una corriente conocida como veriphobia, o cultura de la no verdad, bastante difusa entre los juristas y, en particular, entre los procesalistas (Ureña, 2016, p. 286).

En esencia, dicha perspectiva se cualifica, porque resulta ser a toda costa escéptica frente a "la posibilidad de que se pueda hablar de la verdad en cualquier ámbito posible, y se circunscribe, en el contexto del proceso civil, en la imposibilidad de lograr la determinación verdadera de los hechos" (Taruffo, 2002, p. 27).

De igual forma, la idea de que la jurisdicción es sobre todo instrumento de justicia y su relación con la verdad está dada cuando se acercarse a la verdad sobre los hechos que circundan a las afirmaciones de las partes en el proceso, donde se hace posible hacer justicia en una sociedad democrática organizada como Estado de derecho (Bordalí, 2007, p. 193).

El discurso de la verdad, en un plano ontológico, enlaza con la dimensión epistemológica del proceso, y ha permitido que 
se afirme que la decisión judicial debe abordar con claridad los diversos problemas epistémicos que conlleva todo el proceso. El primero de ellos es el conocimiento del hecho jurídico. La congruencia entre lo representado y lo realmente ocurrido le da solvencia al juez para resolver con argumentos válidos (Ramírez, 2007, p. 176).

Lo anterior demuestra diáfanamente la importancia que se tiene a la hora de señalar con precisión epistemológica la incidencia del activismo procesal, y, con ello, dar cuenta de una teoría coherente y útil en la práctica. No es pensable el proceso, desde dicha ideología, si no se presume desde un inicio que es posible alcanzar la verdad como representación o reconstrucción histórica (Bordalí, 2007, p. 194). Alguna dimensión de la verdad se encuentra llamada a escena en la actuación oficial del juez en el proceso, y aquella enraíza su legitimación, por vía teleológica, en cuanto a la idea de justicia.

Es claro que la verdad guarda una estrecha relación con los hechos y el proceso. Todo ello deriva de la prueba, que es el punto de partida imprescindible para poder tratar adecuadamente el tema atinente a la relación de la verdad de los hechos con el proceso, donde (...) la prueba, (...) conlleva a un sin número de importantes consecuencias de tipo jurídico, entre ellas el papel importante que juega la búsqueda de la verdad de los hechos en el proceso (Ureña, 2016, p. 293).

Observando la proyección de la verdad en el ámbito probatorio, Ferrer (2007), al igual que el profesor italiano Taruffo, coinciden en dos amplios temas alrededor de la prueba: como prueba cerrada y abierta. La primera se identifica con una noción fragmentada de la prueba, y, la segunda, concibe la prueba como un fenómeno ligado a la lógica y la racionalidad, adoptando un punto de vista epistemológico (p. 28).

Desde la perspectiva de Ruiz (2016): 
el derecho se vincula con el conocimiento de los hechos controvertidos y en consecuencia con la verdad, actualizándose su visión de un conjunto de normas de naturaleza procesal encaminadas a conocer esos hechos para resolver en el campo social, una controversia jurídica particular y concreta. Partiendo así de la idea de que esa relación entre derecho y verdad no es igual a aquella que existe entre ésta última y las demás disciplinas científicas. Una de las razones principales de esto es que los elementos constitutivos del derecho son y pertenecen a diversos planos de conocimiento, tal es el caso de los valores, los hechos y las normas (pp. 4-5).

¿Qué es la verdad? ¿Desde donde se construye la verdad? ¿Implica la verdad justicia? ¿Puede haber justicia sin verdad? ¿Es la carga dinámica de la prueba la solución para encontrar la verdad? ¿Solo un juez director - tropos—-puede encontrar la verdad? Las respuestas dependen en gran medida del campo jurídico, como lo expone Bourdieu (citado por Tirado, 2011) en la Fuerza del Derecho.

O como señala Tirado (2011):

El manejo de la verdad históricamente ha sido un problema tanto técnico como político. Técnico porque fundamentalmente las normas procesales (el derecho de acción) han sido establecidas para reglar la forma de llegar a la verdad, que se basa en la prueba y en el mecanismo de dictar justicia. En cualquiera de sus especialidades la actividad jurisdiccional se fundamenta en la manera en que las personas utilizan medios (documentales, testimoniales, periciales, interrogatorios, etc.) para obtener la verdad. (p. 16).

Todo esto se debe valorar siguiendo las reglas de la experiencia, la ciencia y la sana crítica, pues en últimas, la verdad procesal tiene que ver con conocer, con cómo llegar a la verdad (Tirado, 2011, p. 16). Y en cuanto a lo político, la prueba puede variar, modificar y cambiar de forma sustancial en razón de la carga de probar (Tirado, 2011, p. 16).

Lo cierto es que, tal y como lo expresa Ruiz (2016): 
en el trabajo del juez, en pocas controversias jurídicas se logra tener un alto grado de aproximación a la verdad sobre las proposiciones referidas a los hechos que acontecieron en el pasado, y que forman parte de ese conflicto, es decir, tal estado de cosas no debe de llevar a pensar que las demás decisiones judiciales posean un alto grado de imprecisión epistémica, y consecuentemente de subjetividad relatividad, al depender de la voluntad, capacidad y competencias de cada juzgador, puesto que el derecho impone ciertos controles sobre su racionalidad (p. 9).

Esto no quiere decir que los hechos producidos en la fuente de prueba, como ya se ha hablado, hayan sido totalmente reconstruidos en el proceso judicial tal y como sucedieron en el pasado, y con base en esto haya emitido el juez su decisión, pues la tarea epistemológicamente no es nada fácil, de modo que, para ser optimista, el juez, en los casos difíciles, solo puede aspirar a una verdad verosímil de los hechos históricos. En esa lógica argumenta Ruiz (2016):

Las razones técnicas que impiden al juez que pueda conocer la verdad en el proceso son variables; sin embargo, siempre están presentes en todo proceso judicial, pues no pueden ser eliminadas totalmente. Lo único que se puede hacer es controlarlas para así reducir lo más que pueda sus efectos nocivos en la racionalidad de su decisión judicial. En el análisis del tema de la verdad derivada de la prueba no hay que adoptar una posición escéptica o relativista, sino una de tipo objetivista y de corte crítico, en la cual el jurista someta los hechos que le dan a conocer las partes en la controversia jurídica, a un estricto análisis epistemológico, gnoseológico, lógico y dogmático jurídico, complementado con su experiencia, con el fin de determinar hasta qué punto esos hechos son independientes de él, en qué medida son construcciones personales -subjetivas-, y en cuáles casos es posible o hasta qué punto pueden ser conocidos con cierto grado de objetividad (p. 9)

En coherencia con ello Michele Taruffo (2009) agrega: 
Argumentando de esta manera, en el ámbito de la concepción legal-racional de la justicia a la que se ha hecho referencia al principio, se puede concluir que el proceso debe estar orientado hacia la consecución de una decisión verídica, o sea correspondiente en la mayor medida posible con la realidad de los hechos. En este sentido, el proceso puede ser concebido como un procedimiento epistémico, en el que se recogen y se utilizan conocimientos con el objetivo de reconstruir la verdad de determinadas situaciones de hecho (p. 97).

Así las cosas, la verdad procesal es esencialmente relativa, dado que la decisión tomada por el juez en torno a los hechos expuestos por las partes no puede fundamentarse más que en las pruebas que han sido recopiladas durante el curso del proceso. De hecho, las pruebas son el único instrumento mediante el cual un juez puede, en cierta medida, conocer y, por tanto, reconstruir de modo verídico los hechos de la causa.

La circunstancia de que no se hable de verdades absolutas y que la verdad procesal sea "relativa a las pruebas" no pudiendo fundarse más que en ellas, induce a formular el problema de la decisión sobre los hechos, no en términos de certeza sino en términos de probabilidad. Esto sugiere una cuestión ulterior, ya que el concepto de probabilidad no es unívoco, y, por tanto, habría que establecer qué probabilidades entran en juego en el contexto procesal. En otros términos, y como se verá mejor enseguida, el problema de la verdad procesal puede ser correctamente reformulado en términos de grados de confirmación probabilista que las pruebas pueden ofrecer a los enunciados sobre los hechos (Taruffo, 2009, p. 310).

\section{De la verdad procesal a la teoría de la probabilidad}

Uno de los mayores desafíos de la ciencia del derecho probatorio que ha generado incansables discusiones y diversas posiciones teóricas entre la comunidad académica a lo largo de varias décadas hasta los tiempos actuales, es la tensión sobre la certeza absoluta entre la verdad procesal y la verdad material. Si bien en un proceso judicial se tiene por objeto hallar la verdad del caso en controversia y garantizar con esto la protección del bien 
jurídico amenazado o vulnerado, no es menos cierto que en la mayoría de ocasiones dicha verdad es relativa dado que el juez funda el contendido resolutorio de sus decisiones según los resultados arrojados por la valoración de la pruebas practicadas en juicio, es decir, que el único instrumento que tiene el juez para descifrar el acertijo jurídico son los hechos constatados a través de las pruebas ofrecidas en el proceso judicial, que en la generalidad de situaciones no siempre corresponden a la verdad histórica de los acontecimientos.

En otras palabras, debe existir un vínculo causal entre el resultado probatorio y los argumentos expuestos a prueba, lo que permitirá demostrar al juez la verdad de los hechos a la luz de lo "alcanzado-permitido" en el curso del proceso judicial, esto es, la consecución de la verdad procesal, que no es más que la verdad según los resultados condicionados por la prueba, mas no, como no ocurre en muchos casos, "la verdad verdadera" fuera de lo que pudo probarse. Así las cosas, Genuncio Bentini (citado por, Battistelli, 1984), advierte que "la verdad no entra en la sala del Tribunal ni tampoco en pleito celebre alguno. Ella ha quedado siempre en las escaleras o en la calle" (p. 10), por ello no puede negarse que en los procesos se miente para manipular la naturaleza de los hechos, mienten los testigos, los apoderados, los peritos, hasta los fiscales y jueces, es decir, todos los sujetos procesales que intervienen en busca de beneficiar intereses personales o de terceros, raramente alejados de la legalidad, y de esta manera persuadir en las decisiones impartidas por el operador judicial (León, 2003). Al respecto, Lluch (2015), señala:

hay que empezar por señalar que el tema de la verdad como fin del proceso ha preocupado desde hace mucho tiempo a los filósofos del derecho como a los procesalistas, algunos de los cuales piensan que la verdad no puede ser alcanzada en el proceso; mientras para otros, la verdad no debe ser alcanzada en el proceso, pues este tiene por fin la resolución de un conflicto intersubjetivo. Con carácter general puede afirmarse que la verdad siempre es relativa, pues depende tanto de la información suministrada como del método empleado para determinarla (pp. 91-92). 
De tal manera que es en la actividad probatoria, una especie de laboratorio científico, donde se reconstruye la teoría del caso a partir de la comprobación y confirmación de las hipótesis planteadas según los sucesos expuestos en el libelo de la demanda y su contestación. O como afirma Gascón (citada por Peña, 2008), la valoración de la prueba se trata de una actividad racional encaminada a la búsqueda de la hipótesis más acertada entre la universalidad de posibilidades de reconstrucción de los hechos. En igual sentido, Peña (2008) manifiesta que "la valoración de las pruebas corresponde al juicio de aceptabilidad de los resultados derivados de los medios de prueba" (p 44). Esto significa, que valorar es "contrastar enunciados fácticos, para estimar su correspondencia con los hechos que describen; lo cual implica dar cuenta de las razones por las cuales se considera que una alternativa es preferible a otra" (Peña, 2008, p. 44).

Por consiguiente, se colige que solo podrá catalogarse como verdadero aquello que pueda probarse, es decir, que la prueba brinde el suficiente nivel cognoscitivo que permita soportar la veracidad de los hechos, aunque, como se ha dicho anteriormente, esto no siempre corresponde a revelar la verdad de los hechos, ya que según el grado de relatividad de la misma se condicionará el grado de relatividad de la verdad procesal, esto es, que dependerá del medio probatorio y de las pruebas sometidas a este (Montilla y Jiménez, 2016).

En consecuencia, debido a este relativismo propio de la prueba presente en la mayoría de casos, se obstaculiza el trabajo del aparato jurisdiccional para obtener verdades absolutas. Así lo advierte Taruffo (s.f), para el cual, "por un lado, la definición tarskiana del concepto de verdad vale también en el contexto del proceso, por otro lado, hay que destacar que la verdad procesal es esencialmente relativa porque la decisión del juez en torno a los hechos no puede fundarse más que en las pruebas que han sido adquiridas en el juicio" (p. 97).

Empero, frente a esta dificultad del derecho probatorio de obtener verdades absolutas, no se puede descartar la verdad procesal de los hechos, puesto que, ante la ausencia de una verdad perfecta, rechazarla conduciría a que el juez pueda actuar bajo su arbitrio. Por lo tanto, la doctrina ha apelado a 
la búsqueda de la verdad a través de grados de certeza, esto es, de probabilidad, a través del replanteamiento epistemológico de la racionalidad empírica para proporcionar efectos no alejados del objetivismo a través de premisas probables, disminuyendo así el grado de certeza o convicción de los hechos, que, debido a su impedimento de exactitud, resultan lo más probable a la luz de la razón (Ruiz y Velásquez, 2006, pp. 156-157). Al respecto, Gascón (2010) indica:

En concreto, para las nuevas epistemologías empiristas, el objetivo del conocimiento inductivo no es ya la búsqueda de certezas absolutas, sino tan sólo de "supuestos" o hipótesis válidas, es decir, apoyadas por hechos que las hacen "probables". En esto radica su "miseria", pero también su "grandeza": se ha restaurado la confianza en una racionalidad empírica que, renunciando al objetivo inalcanzable de la certeza absoluta, recupera, a través del concepto de "probabilidad", un elemento de objetividad $^{2}$ (p. 12).

Con base en lo señalado antes, la imperfección de la prueba judicial para asegurar la verdad de los hechos, debido en primer plano a las limitaciones y restricciones que devienen de la sistematización de los medios de prueba, subyace sobre esta misma otra razón pero de tipo epistemológico, en la cual "el razonamiento probatorio está constituido básicamente por inferencias inductivas basadas en leyes probabilísticas o incluso por inferencias basadas en generalidades sin demasiado fundamento o sencillamente en prejuicios" (Gascón, 2005, p. 128).

Esto dificultad los resultados de la prueba e impide garantizar la certeza absoluta, y solo posibilita cierto grado de certeza, o de probabilidad, sobre la verdad del enunciado probado (Gascón, 2005, p. 128), de tal forma, que "la verdad de que se habla en el proceso ha de concebirse como aproximación en la reconstrucción procesal de los hechos a su realidad empírica e histórica" (Taruffo, citado por Lluch, 2015, p. 92).

\footnotetext{
${ }^{2}$ Es más, "La recuperación de la racionalidad empírica a través del concepto de probabilidad no carece de consecuencias para la fijación judicial de los hechos" (Gascón, 2010).
} 
Así las cosas, para ilustrar un poco más sobre el tratamiento aplicado a través de las teorías probabilísticas desde enfoques distintos del derecho, se plantea el caso de la sociología, que es, a la final, la ciencia básica de donde parte toda explicación a todo problema estructural. En tal sentido, Niklas Luhmann, unos de los sociólogos más influyentes de finales del siglo XX, abrió la discusión a la "Paradoja de la probabilidad de lo improbable" (Razeto-Barry y Cienfuegos, 2011) como medio para resolver los interrogantes de la evolución biológica y social.

No obstante, ha sido a través de la doctrina que en cierto modo se ha trabajado por responder a ciertos interrogantes de la misma, esto es, "mediante la sistematización de los elementos probabilísticos que caracterizan a los procesos evolutivos, tomando herramientas de la biología evolutiva y la teoría de probabilidades" (Razeto-Barry y Cienfuegos, 2011, p. 13). Esto conduce a plantear que toda base teórica científica se compone de elementos probabilísticos para responder a nuestras necesidades conceptuales.

De modo que, sin ánimo de adentrar exhaustivamente al estudio de otras ciencias, se hace necesario resaltar ciertos aspectos para dar luz y mayor entendimiento a los criterios aquí expuestos (Vargas, 2013, p. 113). ${ }^{3}$ Dos ejemplos ayudan a ilustrar lo dicho por Luhmann en la interpretación de RazetoBarry y Cienfuegos (2011), esto es: "Una constatación probabilísticamente simple, pero muy pocas veces tomada en cuenta, consiste en que la baja probabilidad de que ocurra un evento en una oportunidad particular no limita en absoluto el que su ocurrencia se vuelva altamente probable con el tiempo" (p. 18).

\footnotetext{
${ }^{3}$ Para Vargas (2013), "La obra de Lawrence Jonathan Cohen, Lo probable y lo demostrable, ofrece una visión de la llamada inferencia inductiva, fundamentada en los trabajos de Sir Francis Bacon y John Stuart Mill. La inferencia inductiva conduce a un nuevo concepto de probabilidad que, en palabras de Cohen, congenia mejor con las inferencias que se realizan en el campo jurídico. Cohen aplica lo que denomina la probabilidad inductiva (PI) en el sistema legal anglo-estadounidense y la contrasta con la probabilidad matemática (PM), la cual, a su juicio, es supremamente útil en el estudio de procesos aleatorios y aplicable en diversas áreas científicas, pero insuficiente e inadecuada para las inferencias inductivas que se realizan en el campo jurídico" (p. 113).
} 
Por otra parte, el concepto de probabilidad se le antecede desde los orígenes de la retórica griega, conocido en otros términos como eikóta (Mercado, 2015), que traduce probable o verosímil, concepto que era empleado por los oradores en sus discursos en los tiempos de Isócrates, entre otros, principalmente en los casos en que no se tenían testigos u otras formas de poder demostrar el hecho punible.

Para ello, Mercado (2015) expone una ilustración de la mitología griega: la discusión de Hermes por el ganado de Apolo y los argumentos usados por Hermes en su defensa al objetar que es imposible que un niño recién nacido pueda ser condenado por un hecho que por su condición no puede cometer. Esto es:

Frente a la necesidad de encontrar al verdadero culpable, de impartir justicia, la falta de pruebas concretas enfrenta en igualdad de condiciones la palabra del uno contra la del otro. En esa medida, Apolo se ve en apuros ante un Hermes que apela a la probabilidad para defenderse con el argumento de que es creíble que un niño, que acaba de nacer y que se preocupa más por alimentarse de su madre, no se separe de ella para cometer abigeato. Pero en este caso más que exponer la astucia de Hermes para mentir, Homero habría sentado las bases para la utilización legítima de un recurso que sirve para resolución pacífica y argumentada de complejos conflictos jurídicos (p. 20).

Ahora bien, teniendo ya una idea de la probabilidad en el estudio de otras ciencias, se procede a retomar lo dicho por Gascón (2005) en el acápite sobre la dificultad por parte de la epistemología para lograr garantizar verdades absolutas sino a través de hipótesis soportadas según un grado de probabilidad. Conviene ahora señalar que no basta con que la valoración racional que se haga de las pruebas case con una hipótesis, sino que también es esencial evaluar si ese grado de probabilidad contenido en dicha hipótesis es lo suficiente para sostener su veracidad, esto es el estándar de la prueba, pues ellos son los "criterios que indican cuándo se ha conseguido la prueba de un hecho; o sea, los criterios que indican cuándo está justificado aceptar como verdadera la hipótesis que lo describe” (Gascón, 2005, p. 129).

En cuanto a los estándares de la prueba, Gascón (2005) advierte que comprenden: 
a) en primer lugar implica decidir qué grado de probabilidad o certeza se requiere para aceptar una $\mathrm{H}$ como verdadera.; b) en segundo lugar implica formular objetivamente el SP; es decir, formular los criterios objetivos que indican cuándo se alcanza ese grado de probabilidad o certeza exigido (p. 129).

Para mostrar lo expuesto, el Consejo de Estado de Colombia, en la resolución de sus fallos, ha recurrido a la implementación de argumentos de probabilidad, particularmente en casos de responsabilidad médica donde se ha reducido el estándar o grado de prueba con el objeto de poder establecer la causalidad entre el daño o perjuicio causado y el autor responsable en aquellas situaciones en que por diversas razones se dificulta establecer dicha relación; lo anterior quiere decir que quien reclama el derecho no está en la obligación de demostrar ese vínculo causal, sino que basta con la sola convicción del juez para fundar su decisión sobre una probabilidad preponderante.

Debe anotarse que, antes de que el Estado jurisdicción fundamentara sus decisiones en esta materia bajo los grados de la probabilidad preponderante, se sufrieron varias modificaciones y cambios hasta la evolución actual, pasando desde la teoría de la falla del servicio, el principio de presunción de falla del servicio médico, la teoría de la carga dinámica de la prueba, hasta lo logrado hoy en día (Ruiz y Velásquez, 2006).

Verbigracia de lo dicho, es en la sentencia de fecha 1 de agosto de 2016, Sección Tercera del Consejo de Estado - Sala Plena Contenciosa Administrativa, donde fue ponente el magistrado Carlos Alberto Zambrano Barrera, que se dirimió el caso de un paciente que ingresa a un hospital luego de haber sido víctima de un accidente que le produjo un trauma craneoencefálico hasta producirle la muerte. El paciente recibió atención en dicho centro de salud durante varios días, pero aun así en la historia clínica no se determina el motivo que ocasionó su deceso. El hospital adujo que la atención dada al paciente fue oportuna y que su fallecimiento obedeció a las graves lesiones recibidas y no por la suspensión de los medicamentos y el tipo de alimentación dada, negando la configuración de una falla en la prestación del servicio por negligencia. 
Por su parte, el a quo índico que en la historia clínica no reposaba razón alguna, así como tampoco obra en el plenario un examen de medicina legal que justificara las causas de su deceso, aun así, según el concepto médico de los especialistas consultados se objeta que pudieron ser varias las razones a falta de que en la historia clínica no se indicó nada al respecto, es decir, que se pueden establecer varias posibilidades que conllevaron a acabar con la vida del paciente.

Como resultado, el a quo decide revocar la sentencia de primera instancia y declara la responsabilidad del hospital por pérdida de la oportunidad de salvar su vida, teniendo de presente el siguiente criterio de probabilidad:

Teniendo en cuenta que existía una probabilidad de que el suministro de ranitidina generara en el paciente un cuadro de broncoaspiración, el demandado debió tomar las medidas del caso, a fin de evitar que ello ocurriera. Ahora, si bien a juicio del especialista B.P. el cuadro de broncoaspiración que sufrió el paciente pudo obedecer a diferentes causas, lo cierto es que la historia clínica no las mencionó y, por tanto, se trata de meras hipótesis; por el contrario, está documentado que al señor A.G. se le suministró ranitidina durante toda su estadía en el Hospital Universitario San José de Popayán E.S.E., medicamento que, como se dejó dicho, podía provocar broncoaspiración, cuadro éste que aquél padeció (Consejo de Estado, Sección Tercera, 2016).

\section{Y agrega:}

El juez puede fundar su decisión sobre los hechos que, aun sin estar establecidos de manera irrefutable, aparecen como los más verosímiles, es decir, los que presentan un grado de probabilidad predominante. No basta que un hecho pueda ser considerado sólo como una hipótesis posible. Entre los elementos de hecho alegados, el juez debe tener en cuenta los que le parecen más probables. Esto significa sobre todo que quien hace valer su derecho fundándose en la relación de causalidad natural entre un suceso y un daño, no está obligado a demostrar esa relación con exactitud científica. Basta con que el juez, en el caso en que por la naturaleza de las cosas no cabe una prueba directa, llegue a la convicción de que existe una "probabilidad determinante" (Consejo de Estado, Sección Tercera, 2016). 


\section{Conclusiones}

Se establece en este trabajo que la carga cognoscitiva de la prueba es la que permite justificar la reconstrucción de la verdad de los hechos, es decir, sin hecho no hay duda que no hay prueba, pues esta es el fundamento del hecho. Junto a esto, se resalta la comprensión de la prueba desde la adecuada distinción entre fuente, medio y objeto de prueba.

También se demostró que no tiene valor alguno una distinción entre fuente, medio y objeto si se omite la responsabilidad del protagonista de la valoración probatoria: el juez, pues la prueba por sí sola no habla, se necesita de un intérprete que haga posible que la prueba despliegue una carga de garante de los derechos humanos para reconstruir la verdad fáctica o por correspondencia de lo que pasó.

La valoración de la prueba quedó manifestada como una actividad fuerte del engranaje epistemológico del Derecho que emerge como consecuencia de hechos del pasado que tienen el carácter de ser jurídicamente relevantes, pero con la dificultad de ser hechos que desconocen los juristas. No obstante, a través de la estructura de los medios de prueba, se hace posible la reconstrucción de la verdad histórica de los hechos. Verdad que no puede ser absoluta, solo es la verdad conforme al proceso, de ahí que lo único seguro es que es relativa y responde al material probatorio aportado par las partes y al juez si tiene poderes oficiosos.

Por su parte, la verdad procesal o verdad judicial, también conocida como verdad relativa, existe y se reconoce de acuerdo con los aportes probatorios de parte o por los instrumentos oficiosos del Estado jurisdicción en aquellos sistemas jurídicos donde aún existen o donde, a pesar de $\mathrm{su}$ inexistencia, los jueces son jueces Hércules o jueces Tropos, es decir, directores del proceso con capacidades plenas para alcanzar la verdad. De ahí, como se demostró, la verdad existe independiente de los grados de confirmación procesal. 
En otras palabras, la verdad procesal es cognoscitiva, de modo que la verdad de los hechos es posible, al menos de los hechos históricos que se trasladan al proceso, por esta razón, como se demostró, la importancia misional de la prueba como fuente, medio y objeto resultan clave, pues cada una de ellas cumple en detalle un fin con relación a la verdad de los hechos y, en consecuencia, no es posible una verdad de los hechos en abstracto, pues la verdad, así sea relativa, requiere de la especificidad de la ocurrencia de los hechos.

De otra parte, debido a la constante dificultad de los sistemas jurisdiccionales de develar la verdad histórica de los casos en juicio, la probabilidad preponderante permite encontrar una luz a esa falencia a través de la reducción de los estándares de prueba, pero ceñidos a una estricta y objetiva valoración de la prueba de los grados de probabilidad que se requiere para aceptar como verdadera una hipótesis. Esto con el fin de garantizar el efectivo cumplimiento de hacer justicia y no dejar en la injusticia o impunidad hechos difíciles de probar.

Por último, la probabilidad preponderante cada día se hará más fuerte en los esquemas procesales, las dinámicas de la ciencia y la tecnología permitirán que los hechos jurídicamente relevantes sean medidos y, con ello, calcular las formas y modos de ocurrencia de hechos futuros en la valoración que realizan los jueces. En este sentido, los cálculos de probabilidad no son del azar, requieren de un buen interprete, o sea, de un buen juez capaz de calcular probabilidades.

\section{Referencias}

Acosta, L. (2007). Diferencias entre medio, fuente y objeto de la prueba. Cuestiones jurídicas, 1(2), 51-72.

Battistelli, L. (1984). La mentira ante los tribunales: Fragmentos y apuntes de sicología y sicopatología judicial. Bogotá, D.C.: Editorial Temis. 
Bordalí, A. (2007). Los poderes del juez civil. Proceso civil. Hacia una nueva justicia civil, 179.

Consejo de Estado, Sala Plena Contenciosa Administrativa Sección Tercera. (1 de agosto de 2016). Sentencia N ${ }^{\circ} 19001-$ 23-31-000-2001-01429-01 de 1 de agosto de 2016. [C. P. Carlos Alberto Zambrano Barrera].

Ferrer, J. (2007). La valoración racional de la prueba. Madrid: Marcial Pons.

Gascón, M. (2010). Los hechos en el derecho. Bases argumentales de la prueba. Madrid: Marcial Pons.

Gascón, M. (2005). Sobre la posibilidad de formular estándares de prueba objetivos. DOXA Cuadernos de Filosofía del Derecho, 28.

León, A. (2003). Breve tratado sobre la mentira. Colección Artes y Humanidades filosofía. Cali: Editorial Universidad del Valle.

Lluch, X. (2015). Estándares de prueba y reglas de la sana crítica. En: Derecho procesal y perspectiva científica de la prueba. Barranquilla: Editorial Universidad Simón Bolívar.

Meneses, C. (2008). Fuentes de prueba y medios de prueba en el proceso civil. Ius et Praxis, 14 (2), 1-36.

Mercado, H. (2015). El argumento de probabilidad (tòcìkós) en la retórica griega. Revista Perseitas 3(1). 13-33.

Montilla, C. y Jiménez, J. (2016). Alcance y limitaciones de la carga dinámica de la prueba en el proceso judicial. Medellín: Universidad de Medellín.

Peña, J. (2008). Prueba judicial, análisis y valoración. Edición Escuela Judicial Rodrigo Lara Bonilla - C.S. de la J, Sala Administrativa.

Ramírez, D. (2007). A propósito de la justicia material: Reflexiones sobre la justicia en el proceso versus la justicia material. Disponible en http:// redalyc.uaemex.mx/redalyc/src/inicio/ ArtPdfRed.jsp?iCve $=94501209$

Razeto-Barry, P. y Cienfuegos, J. (2011). La paradoja de la probabilidad de lo improbable y el pensamiento evolutivo de Niklas Luhmann. Convergencia. Revista de Ciencias Sociales, 18(57), 13-38. 
Rodríguez, F. (2009). Tres imperativos categóricos para una correcta decisión judicial. Jurid. Manizales (Colombia), 6(1), 69-78. Disponible en: http://132.248.9.34/hevila/JuridicasManizales/2009/vol6/no1/4.pdf

Rodríguez, F. y Ospino, S. (2009). El juez tropos en el marco del Estado Social de Derecho y la garantía al debido proceso. Revista Justicia, 16, 53-61. Disponible en: http://revistas. unisimon.edu.co/index.php/justicia/article/view/627/615

Ruiz, J. A. (2016). La verdad en el Derecho. Intersticios sociales, (12), 1-33.

Ruiz, L. (2007). El derecho a la prueba como un derecho fundamental. Estudios de Derecho, 64(143), 181-206.

Ruiz, L. y Velázquez, R. (2006). Valoración de la prueba en la responsabilidad por la actividad médica, concepto de probabilidad y las garantías procesales. Medellín: Zenit.

Taruffo, M. (2009). La prueba, artículos y conferencias. Santiago de Chile: Metropolitana.

Taruffo, M. (2008). La prueba. Madrid: Marcial Pons.

Taruffo, M. (2005). Conocimiento científico y estándares de prueba judicial. Boletín Mexicano de Derecho Comparado, 38(114), 1285-1312.

Taruffo, M. (2002). La prueba de los hechos. Jordi Ferrer Beltrán (trad.). Madrid: Trotta.

Taruffo, M. (s.f). La prueba, Artículos y Conferencias”, Monografías Jurídicas Universitas, Editorial Metropolitana.

Tirado, M. (2011). Verdad, prueba e indagación en el mundo del Derecho y de la sociedad. Prolegómenos, 14(27), 13-26.

Vargas, (2013). Lo Probable y lo Demostrable. Una Aproximación a la Obra de Lawrence Jonathan Cohen. Revista Academia \& Derecho, 6(4). 111-124. https://doi.org/10.18041/2215-8944/ academia.6.2468

Vargas, R. (2011). Concepciones de la prueba judicial. Prolegómenos, 14(28), 135-148.

Vargas, R. (2010). Valoración de la prueba científica de ADN en el proceso penal. Prolegómenos, 13(25), 127-146. 
Vega, H. (2015). Aspectos dogmáticos y políticos criminales de la estructura general del delito en el sistema penal colombiano. Justicia, 27, 42-72.

Verdugo, M. (2010). Declaración de la parte como medio de prueba. Ius et Praxis, 16(1), 125-170.

Ureña, B. (2016). La verdad de los hechos como conditio sine qua non de una decisión judicial justa en el pensamiento de Michele Taruffo. Boletín mexicano de derecho comparado, 49(146), 281-304. 
Ferney Asdrúbal Rodríguez-Serpa es Doctor en Filosofía con orientación en Ciencias Políticas de la Universidad Autónoma de Nuevo León de Monterrey-México; magíster en Derecho Procesal; abogado; sociólogo; editor de la Revista Justicia de la Universidad Simón Bolívar, Colombia; docente-investigador vinculado al grupo de investigación científica "Derechos humanos, tendencias jurídicas y sociojuridicas contemporáneas" de la Universidad Simón Bolívar de Barranquilla, Colombia..

Nelhiño Bolaño Miranda es Magíster en Derecho Procesal de la Universidad Simón Bolívar de Barranquilla. Abogado, especialista en Derecho Público de la Universidad del Norte. Investigador externo asociado al grupo de investigación científica "Derechos humanos, tendencias jurídicas y sociojuridicas contemporáneas" de la Universidad Simón Bolívar de Barranquilla, Colombia.

Gloria María Algarín Herrera es Abogada de la Universidad Simón Bolívar de Barranquilla, experta en Derechos Humanos y Derecho Internacional de los Derechos Humanos, y litigio estratégico ante la comisión interamericana de DD. HH y la Corte Interamericana de DD. HH. Investigadora externa asociada al grupo de investigación científica "Derechos humanos, tendencias jurídicas y sociojuridicas contemporáneas" de la Universidad Simón Bolívar de Barranquilla, Colombia. 